[VoL. IX.

\title{
ON A THEOREM OF CLEBSCH'S.
}

By Paul Saurel.

LEBSCH has shown' that the integrals of the equations of motion of a vibrating elastic solid can be put into a very simple and elegant form. His demonstration, while by no means long or difficult, seems, nevertheless, to admit of some simplification.

The equations in question are $:^{2}$

$$
\begin{aligned}
\frac{\partial^{2} u}{\partial t^{2}} & =a^{2} \Delta u+\left(b^{2}-a^{2}\right) \frac{\partial \sigma}{\partial x} \\
\frac{d^{2} v}{\partial t^{2}} & =a^{2} \Delta v+\left(b^{2}-a^{2}\right) \frac{\partial \sigma}{\partial y} \\
\frac{\partial^{2} w}{\partial t^{2}} & =a^{2} \Delta w+\left(b^{2}-a^{2}\right) \frac{\partial \sigma}{\partial z} \\
\Delta & =\frac{\partial^{2}}{\partial x^{2}}+\frac{\partial^{2}}{\partial y^{2}}+\frac{\partial^{2}}{\partial z^{2}} \\
\sigma & =\frac{\partial u}{\partial x}+\frac{\partial v}{\partial y}+\frac{\partial w}{\partial z} .
\end{aligned}
$$

in which

and

From the above equations, it follows without difficulty that

$$
\frac{\partial^{2} \sigma}{\partial t^{2}}=b^{2} \Delta \sigma
$$

Let $u, v, w$ be any given solution of the above equations and let us write

$$
\left.\begin{array}{c}
u=\frac{\partial P}{\partial x}+u^{\prime} \\
v=\frac{\partial P}{\partial y}+v^{\prime} \\
w+\frac{\partial P}{\partial z}+w^{\prime}
\end{array}\right\} .
$$

${ }^{1}$ Clebsch, Ueber die Reflexion an einer Kugelfäche. Borchardt's Journal für die reine $u$. angew Math., Bd. 6r.

${ }^{2}$ Kirchhoff, Vorlesungen über Mechanik, XI Vorlesung. 
From these we obtain

$$
\sigma=\Delta P+\sigma^{\prime}
$$

in which

$$
\sigma^{\prime}=\frac{\partial u^{\prime}}{\partial x}+\frac{\partial v^{\prime}}{\partial y}+\frac{\partial w^{\prime}}{\partial z}
$$

If we substitute the above values of $u, v, w$ in the equations of motion, we get

$$
\left.\begin{array}{l}
\frac{\partial^{2} u^{\prime}}{\partial t^{2}}+\frac{\partial}{\partial x}\left[\frac{\partial^{2} P}{\partial t^{2}}-b^{2} \Delta P\right]=a^{2} \Delta u^{\prime}+\left(b^{2}-a^{2}\right) \frac{\partial \sigma^{\prime}}{\partial x} \\
\frac{\partial^{2} v^{\prime}}{\partial t^{2}}+\frac{\partial}{\partial y}\left[\frac{\partial^{2} P}{\partial t^{2}}-b^{2} \Delta P\right]=a^{2} \Delta v^{\prime}+\left(b^{2}-a^{2}\right) \frac{\partial \sigma^{\prime}}{\partial y} \\
\frac{\partial^{2} w^{\prime}}{\partial t^{2}}+\frac{\partial}{\partial z}\left[\frac{\partial^{2} P}{\partial t^{2}}-b^{2} \Delta P\right]=a^{2} \Delta z w^{\prime}+\left(b^{2}-a^{2}\right) \frac{\partial \sigma^{\prime}}{\partial z}
\end{array}\right\}
$$

Suppose that

$$
\sigma^{\prime}=0 \text {. }
$$

The necessary and sufficient condition for this, is (equation 4 ) that

$$
\Delta P=\sigma .
$$

Suppose moreover that $P$ satisfies the equation

$$
\frac{\partial^{2} P}{\partial t^{2}}=b^{2} \Delta P
$$

Equations 5 then become

$$
\left.\begin{array}{l}
\frac{\partial^{2} u^{\prime}}{\partial t^{2}}=a^{2} \Delta u^{\prime} \\
\frac{\partial^{2} v^{\prime}}{\partial t^{2}}=a^{2} \Delta v^{\prime} \\
\frac{\partial^{2} w^{\prime}}{\partial t^{2}}=a^{2} \Delta w
\end{array}\right\}
$$

If, then, it be possible to choose $P$ so that equations 7 and 8 are simultaneously verified, equations 3 will determine $u^{\prime}, v^{\prime}, w^{\prime}$. We shall thus have established that every solution of the given equations can be written in the form of equations $3, P$ being a common integral of equations 7 and 8 , and $\iota^{\prime}, v^{\prime} w w^{\prime}$ being three solutions of the equation

$$
\frac{\partial^{2} \varphi}{\partial t^{2}}=a^{2} \Delta \varphi
$$


these solutions, moreover, being connected by equation 6 :

$$
\frac{\partial u^{\prime}}{\partial x}+\frac{\partial v^{\prime}}{\partial y}+\frac{\partial w^{\prime}}{\partial z}=0 \text {. }
$$

In order to show that we can determine $P$ so as to satisfy equations 7 and 8 , let us write the equation obtained from them :

$$
\frac{\partial^{2} P}{\partial t^{2}}=b^{2} \sigma
$$

The pair of equations 7 and 8 may be replaced by the pair of equivalent equations 7 and 12 . The integral of equation 12 is

$$
P=b^{2} \int d t \int \sigma d t+A(x, y, z) \cdot t+B(x, y, z),
$$

$A$ and $B$ being two arbitrary functions. From this equation we obtain

$$
\Delta P=b^{2} \int d t \int \Delta \sigma d t+\Delta A(x, y, z) \cdot t+\Delta B(z, y, z) .
$$

Choosing the arbitrary functions so that

$$
\Delta A(x, y, z)=0, \quad \Delta B(x, y, z)=0
$$

and making use of equation 2 , we obtain

$$
\Delta P=\int d t \int \frac{\partial^{2} \sigma}{\partial t^{2}} d t=\sigma
$$

Thus equation 7 is also verified and the theorem stated above is established.

This result may be put into a more striking form if we make use of a theorem tacitly admitted by Clebsch and rigorously established by Duhem. ${ }^{1}$ The theorem in question is the following: If $u^{\prime}, v^{\prime}$, $w^{\prime}$ are three integrals of equation IO, which also verify equation II, we may write

$$
\begin{aligned}
u^{\prime} & =\frac{\partial W}{\partial y}-\frac{\partial V}{\partial z}, \\
v^{\prime} & =\frac{\partial U}{\partial z}-\frac{\partial W}{\partial x}, \\
w^{\prime} & =\frac{\partial V}{\partial x}-\frac{\partial U}{\partial y},
\end{aligned}
$$

in which $U, V, W$ are three solutions of equation Io.

1 Duhem, Sur l'intégrale des équations des petits mouvements d'un solide isotrope. Mémoires de la Société des Sciences de Bordeaux. $5^{\ominus}$ Série, t. III., p. 325, I899. 
Thus finally we obtain Clebsch's theorem : Every solution of the equations of motion of a vibrating elastic solid can be written in the form :

$$
\left.\begin{array}{r}
u=\frac{\partial P}{\partial x}+\frac{\partial W}{\partial y}-\frac{\partial V}{\partial z} \\
v=\frac{\partial P}{\partial y}+\frac{\partial U}{\partial z}-\frac{\partial W}{\partial x} \\
w=\frac{\partial P}{\partial z}+\frac{\partial V}{\partial x}-\frac{\partial U}{\partial y}
\end{array}\right\},
$$

$U, V, W$ being three solutions of the equation

$$
\frac{\partial^{2} \varphi}{\partial t^{2}}=a^{2} \Delta \varphi
$$

and $P$ being a solution common to the equations

$$
\begin{gathered}
\frac{\partial^{2} P}{\partial t^{2}}=b^{2} \Delta P \\
\Delta P=\sigma .
\end{gathered}
$$

Conversely, if $U, V, W$ are any three solutions of equation 14, and $P$ any solution of equation I 5 , equations I 3 furnish a solution of the equations of motion. This is very easily verified by substituting in the original equations the values of $u, v, w$ given by equations $\mathrm{I} 3$.

BORDEAUX, April, I899. 